\title{
Value distribution of meromorphic functions concerning rational functions and differences
}

\author{
Mingliang Fang ${ }^{1}$, Degui Yang ${ }^{2}$ and Dan Liu ${ }^{2 *}$ (D)
}

${ }^{\text {*Correspondence: }}$

liudan@scau.edu.cn

${ }^{2}$ Institute of Applied Mathematics, South China Agricultural University,

Wushan Road, Guangzhou, China

Full list of author information is

available at the end of the article

\begin{abstract}
Let $c$ be a nonzero constant and $n$ a positive integer, let $f$ be a transcendental meromorphic function of finite order, and let $R$ be a nonconstant rational function. Under some conditions, we study the relationships between the exponent of convergence of zero points of $f-R$, its shift $f(z+n c)$ and the differences $\Delta_{c}^{n} f$.
\end{abstract}

MSC: Primary 30D35; secondary 39B32

Keywords: Meromorphic functions; Value distribution; Rational functions; Differences

\section{Introduction and main results}

Nevanlinna theory is one of the most important developments in complex analysis in the 20th century, which deals with the value distribution of entire and meromorphic functions. Many applications can be found in the study of Picard type theorems, value sharing results and normality criteria.

In this paper, we assume that the reader is familiar with the basic notations of Nevanlinna's value distribution theory (see [11, 15-18]). In the following, a meromorphic function always means meromorphic in the whole complex plane. By $S(r, f)$, we denote any quantity satisfying $S(r, f)=o(T(r, f))$ as $r \rightarrow \infty$ possible outside of an exceptional set $E$ with finite logarithmic measure $\int_{E} d r / r<\infty$. A meromorphic function $a$ is said to be a small function of $f$ if it satisfies $T(r, a)=S(r, f)$.

Let $f$ be a nonconstant meromorphic function. The order of $f$ is defined by

$$
\rho(f)=\varlimsup_{r \rightarrow \infty} \frac{\log ^{+} T(r, f)}{\log r} .
$$

The exponents of convergence of zeros and poles of $f$ are defined by

$$
\lambda(f)=\varlimsup_{r \rightarrow \infty} \frac{\log ^{+} N\left(r, \frac{1}{f}\right)}{\log r}
$$

and

$$
\lambda\left(\frac{1}{f}\right)=\varlimsup_{r \rightarrow \infty} \frac{\log ^{+} N(r, f)}{\log r} .
$$

(c) The Author(s) 2020. This article is licensed under a Creative Commons Attribution 4.0 International License, which permits use, sharing, adaptation, distribution and reproduction in any medium or format, as long as you give appropriate credit to the original author(s) and the source, provide a link to the Creative Commons licence, and indicate if changes were made. The images or other third party material in this article are included in the article's Creative Commons licence, unless indicated otherwise in a credit line to the material. If material is not included in the article's Creative Commons licence and your intended use is not permitted by statutory regulation or exceeds the permitted use, you will need to obtain permission directly from the copyright holder. To view a copy of this licence, visit http://creativecommons.org/licenses/by/4.0/. 
For a nonzero complex constant $\eta \in \mathbb{C}$, we define the difference operators of $f$ as $\triangle_{\eta} f(z)=f(z+\eta)-f(z)$ and $\triangle_{\eta}^{n} f(z)=\triangle_{\eta}\left(\triangle_{\eta}^{n-1} f(z)\right), n \in \mathbb{N}, n \geq 2$ (see [7, 8, 12-14]).

Recently, numerous mathematicians have studied fixed points, which is an important topic in the theory of meromorphic functions (see [3-6, 9, 10]). In 2000, Chen [4] studied fixed points of solutions of differential equations and defined the exponent of convergence of fixed points by $\tau(f)$ as follows:

$$
\tau(f)=\varlimsup_{r \rightarrow \infty} \frac{\log ^{+} N\left(r, \frac{1}{f-z}\right)}{\log r} .
$$

In 2000, Fang [10] studied the fixed points of derivative of meromorphic functions and obtained the following result.

Theorem A Letf be a transcendental meromorphic function. Suppose that all zeros and poles off are multiple. Then $f^{\prime}$ has infinitely many fixed points.

The topic on fixed points can be also investigated in the field of complex differences. For example, Chen and Shon [6] have got some results on the zeros and fixed points of transcendental entire functions and meromorphic functions. Chen [5] and Zhang-Chen [19] studied the relationships between fixed points of meromorphic functions and their differences and shifts. Their results are stated as follows.

Theorem B ([5]) Let $f$ be a finite order meromorphic function such that $\lambda\left(\frac{1}{f}\right)<\rho(f)$, and let $c(\neq 0)$ be a finite constant such that $\Delta_{c} f(z) \not \equiv c$. Then

$$
\begin{aligned}
& \max \left\{\tau(f(z)), \tau\left(\Delta_{c} f(z)\right)\right\}=\rho(f), \\
& \max \{\tau(f(z)), \tau(f(z+c))\}=\rho(f), \\
& \max \left\{\tau\left(\Delta_{c} f(z)\right), \tau(f(z+c))\right\}=\rho(f) .
\end{aligned}
$$

Theorem C ([19]) Let a be a finite complex number, let $f$ be a finite order meromorphic function such that $\lambda(f-a)<\rho(f)$, and let $c(\neq 0)$ be a finite constant. Then

$$
\begin{aligned}
& \max \left\{\tau(f(z)), \tau\left(\Delta_{c} f(z)\right)\right\}=\rho(f), \\
& \max \{\tau(f(z)), \tau(f(z+c))\}=\rho(f), \\
& \max \left\{\tau\left(\Delta_{c} f(z)\right), \tau(f(z+c))\right\}=\rho(f) .
\end{aligned}
$$

Chen and Zheng [3] extended Theorem C and proved the following theorem.

Theorem D ([3]) Let a be a finite complex number, let $f$ be a finite order transcendental meromorphic function such that $\lambda(f-a)<\rho(f)$, and let $c(\neq 0)$ be a finite constant and $n$ be a positive integer. Then

$$
\begin{aligned}
& \max \left\{\tau(f(z)), \tau\left(\Delta_{c}^{n} f(z)\right)\right\}=\rho(f), \\
& \max \{\tau(f(z)), \tau(f(z+n c))\}=\rho(f), \\
& \max \left\{\tau\left(\Delta_{c}^{n} f(z)\right), \tau(f(z+n c))\right\}=\rho(f) .
\end{aligned}
$$


They generalized the case of fixed points into the more general case for $n=1$, and obtained the following result.

Theorem $\mathbf{E}$ ([3]) Let a be a finite complex number, let $f$ be a finite order transcendental meromorphic function such that $\lambda(f-a)<\rho(f)$, let $c(\neq 0)$ be a finite constant and $m$ be a positive integer, and let $p(z)=p_{m} z^{m}+p_{m-1} z^{m-1}+\cdots+p_{1} z+p_{0}$ be a nonconstant polynomial, where $p_{m} \neq 0, p_{m-1}, \ldots, p_{1}, p_{0}$ are finite complex numbers. Then

$$
\begin{aligned}
& \max \left\{\lambda(f(z)-p(z)), \lambda\left(\Delta_{c} f(z)-p(z)\right)\right\}=\rho(f), \\
& \max \{\lambda(f(z)-p(z)), \lambda(f(z+c)-p(z))\}=\rho(f), \\
& \max \left\{\lambda\left(\Delta_{c} f(z)-p(z)\right), \lambda(f(z+c)-p(z))\right\}=\rho(f) .
\end{aligned}
$$

In 2003, Bergweiler and Pang [2] extended Theorem A as follows.

Theorem $\mathbf{F}$ Let $f$ be a transcendental meromorphic function and let $R(\not \equiv 0)$ be a rational function. Suppose that all but finitely many zeros and poles of $f$ are multiple. Then $f^{\prime}-R$ has infinitely many zeros.

By the above theorems, we naturally pose the following question.

Problem 1 In Theorem B-Theorem E, whether the fixed points or the nonconstant polynomial $p$ can be replaced by a nonconstant rational function $R$ ? In this paper, we give a positive answer to Problem 1 and prove the following results.

Theorem 1 Let $a, c(\neq 0)$ be two finite complex numbers, let $f$ be a finite order transcendental meromorphic function such that $\lambda(f-a)<\rho(f)$, and let $R$ be a nonconstant rational function. Then

(1.1) $\max \left\{\lambda(f(z)-R(z)), \lambda\left(\Delta_{c}^{n} f(z)-R(z)\right)\right\}=\rho(f)$;

(1.2) $\max \{\lambda(f(z)-R(z)), \lambda(f(z+n c)-R(z))\}=\rho(f)$;

(1.3) $\max \left\{\lambda\left(\Delta_{c}^{n} f(z)-R(z)\right), \lambda(f(z+n c)-R(z))\right\}=\rho(f)$.

(1.1), (1.3) are valid unless $f(z)=a+\frac{a-R(z)}{D e^{A z}-1}$, where $A, D$ are nonzero constants, $R$ is a polynomial of $1 \leq \operatorname{deg} R<n$, and $e^{A c}=1$.

Remark Let $n$ be a positive integer, $a, c(\neq 0)$ be two finite values with $e^{c}=1$, let $f(z)=$ $a+\frac{a-R(z)}{e^{z}-1}$, and let $R$ be a polynomial of $1 \leq \operatorname{deg} R<n$. Then $\Delta_{c}^{n} f \equiv 0$. But (1.1) and (1.3) are not valid. Let $R(z)=z, n \geq 2$, in this case, Theorem $\mathrm{D}$ is not valid.

The following two examples show that it is necessary that $R$ is nonconstant.

Example 1 Let $a, R(\neq 0, a), c(\neq 0)$ be three finite complex numbers with $e^{c}=1$, let $n$ be a positive integer, and let $f(z)=a+\frac{a-R}{e^{z}-1}$. Then $f(z) \neq a, f(z) \neq R, f(z+n c) \neq R, \Delta_{c}^{n} f \equiv 0 \neq R$.

Example 2 Let $a, c$ be two nonzero finite complex numbers with $e^{c}=2$, let $R=0$, and let $f(z)=\frac{a}{1-e^{z}}$. Then $\Delta_{c} f=\frac{a e^{z}}{\left(1-e^{z}\right)\left(1-2 e^{z}\right)}, f \neq a, f \neq R, \Delta_{c} f \neq R$.

Theorem 2 Let $c(\neq 0)$ be a finite constant, let $f$ be a finite order transcendental meromorphic function such that $\lambda\left(\frac{1}{f}\right)<\rho(f)$, and let $R$ be a nonconstant rational function. If $\Delta_{c}^{n} f$ is a transcendental meromorphic function, then 
(2.1) $\max \left\{\lambda(f(z)-R(z)), \lambda\left(\Delta_{c}^{n} f(z)-R(z)\right)\right\}=\rho(f)$;

(2.2) $\max \{\lambda(f(z)-R(z)), \lambda(f(z+n c)-R(z))\}=\rho(f)$;

(2.3) $\max \left\{\lambda\left(\Delta_{c}^{n} f(z)-R(z)\right), \lambda(f(z+n c)-R(z))\right\}=\rho(f)$.

Example 3 Let $R$ be a rational function, let $f(z)=e^{z}+R(z)$, and let $c$ is a nonzero constant with $e^{c}=1$. Then $\Delta_{c} f(z)=\Delta_{c} R(z)$. Obviously, $\rho(f)=1, \lambda(f(z)-R(z))=0, \lambda\left(\Delta_{c} f(z)-R(z)\right)=$ $0, \lambda(f(z+c)-R(z))=0$. Thus we obtain $\max \left\{\lambda(f(z)-R(z)), \lambda(f(z+c)-R(z)), \lambda\left(\Delta_{c} f(z)-\right.\right.$ $R(z))\}<\rho(f)$.

This shows that it is necessary that $\Delta_{c}^{n} f$ is a transcendental meromorphic function in Theorem 2.

\section{Some lemmas}

For the proof of our results, we need the following lemmas.

Lemma $1([7,8,13])$ Let $f$ be a meromorphic function of finite order, let c be a nonzero finite complex number. Then

$$
m\left(r, \frac{f(z+c)}{f(z)}\right)=S(r, f),
$$

and, for any $\varepsilon>0$, we have

$$
m\left(r, \frac{f(z+c)}{f(z)}\right)=O\left(r^{\rho(f)-1+\varepsilon}\right) .
$$

Lemma $2([16,17])$ Let $f$ be a meromorphic function. Then, for all irreducible rational functions in $f$,

$$
R(z, f)=\frac{\sum_{i=0}^{m} a_{i}(z) f^{i}}{\sum_{j=0}^{n} b_{j}(z) f^{j}}
$$

with meromorphic coefficients $a_{i}(z), b_{j}(z)$ such that

$$
\begin{array}{ll}
T\left(r, a_{i}\right)=S(r, f), & i=0,1, \ldots, m, \\
T\left(r, b_{j}\right)=S(r, f), & j=0,1, \ldots, n,
\end{array}
$$

the characteristic function of $R(z, f)$ satisfies

$$
T(r, R(z, f))=\max \{m, n\} T(r, f)+S(r, f) .
$$

Lemma 3 ([7]) Let $f$ be a meromorphic function of finite order, let c be a nonzero finite complex number. Then

$$
\begin{aligned}
& T(r, f(z+c))=T(r, f)+S(r, f), \\
& N(r, f(z+c))=N(r, f)+S(r, f), \\
& N\left(r, \frac{1}{f(z+c)}\right)=N\left(r, \frac{1}{f}\right)+S(r, f) .
\end{aligned}
$$


Lemma 4 ([15-18]) Let $f$ be a nonconstant meromorphic function, and let $a_{1}, a_{2}, a_{3}$ (one may be $\infty$ ) be three distinct small functions of $f$. Then

$$
T(r, f) \leq \bar{N}\left(r, \frac{1}{f-a_{1}}\right)+\bar{N}\left(r, \frac{1}{f-a_{2}}\right)+\bar{N}\left(r, \frac{1}{f-a_{3}}\right)+S(r, f) .
$$

Lemma 5 Let $R$ be a nonconstant rational function, let c be a nonzero constant, and let $n$ be a positive integer. Then $\Delta_{c}^{n} R-R \not \equiv 0$.

Proof We consider two cases.

Case 1. $R$ is a nonconstant polynomial. Then $\Delta_{c}^{n} R$ is a polynomial of degree $\operatorname{deg} R-n$ for $n \leq \operatorname{deg} R$, and $\Delta_{c}^{n} R \equiv 0$ for $n>\operatorname{deg} R$. It follows that $\Delta_{c}^{n} R-R \not \equiv 0$.

Case 2. $R=q / p$, where $p, q$ are two coprime polynomials with $\operatorname{deg} p \geq 1$. Set

$$
p(z)=a_{s} z^{s}+\cdots+a_{1} z+a_{0}, \quad q(z)=b_{t} z^{t}+\cdots+b_{1} z+b_{0},
$$

where $a_{0}, a_{1}, \ldots, a_{s}, b_{0}, b_{1}, \ldots, b_{t}$ are finite complex numbers with $a_{s} b_{t} \neq 0$.

Thus we have

$$
\begin{aligned}
\Delta_{c}^{n} R-R & =\frac{q(z+n c)}{p(z+n c)}+\cdots+(-1)^{n-1} C_{n}^{n-1} \frac{q(z+c)}{p(z+c)}+(-1)^{n} \frac{q(z)}{p(z)}-\frac{q(z)}{p(z)} \\
& =\frac{-\left(a_{s}\right)^{n} b_{t} z^{n s+t}+c_{1} z^{n s+t-1}+\cdots+c_{n s+t}}{p(z) p(z+c) \cdots p(z+n c)} \not \equiv 0 .
\end{aligned}
$$

Lemma 6 Let $n$ be a positive integer, let $c$ be a nonzero constant, and let $R=q / p$ be a nonconstant rational function, where $p, q$ are two coprime polynomials. If $\operatorname{deg} p \geq 1$, or $R$ is a polynomial with $\operatorname{deg} R \geq n$, then $\Delta_{c}^{n} R \not \equiv 0$.

Proof We consider two cases.

Case 1. $R$ is a nonconstant polynomial with $\operatorname{deg} R \geq n$. Set $\operatorname{deg} R=s \geq n$, then we have

$$
R(z)=a_{s} z^{s}+\cdots+a_{1} z+a_{0},
$$

where $a_{0}, a_{1}, \ldots, a_{s}$ are finite complex numbers with $a_{s} \neq 0$. Then

$$
\begin{aligned}
\Delta_{c} R= & {\left[a_{s}(z+c)^{s}+\cdots+a_{1}(z+c)+a_{0}\right]-\left[a_{s} z^{s}+\cdots+a_{1} z+a_{0}\right] } \\
= & s a_{s} c z^{s-1}+\cdots, \\
\Delta_{c}^{2} R= & \Delta_{c}\left(\Delta_{c} R\right)=s(s-1) a_{s} c^{2} z^{s-2}+\cdots, \\
& \cdots \\
\Delta_{c}^{n} R= & \Delta_{c}\left(\Delta_{c}^{n-1} R\right)=s(s-1) \cdots(s-n+1) a_{s} c^{n} z^{s-n}+\cdots \neq \equiv .
\end{aligned}
$$

Case 2. $R=q / p$, where $p, q$ are two coprime polynomials with $\operatorname{deg} p \geq 1$. Then we claim that

$$
\Delta_{c} R(z)=\frac{q(z+c)}{p(z+c)}-\frac{q(z)}{p(z)}=\frac{p(z) q(z+c)-p(z+c) q(z)}{p(z) p(z+c)}=\frac{q_{1}(z)}{p_{1}(z)} \not \equiv 0,
$$

where $p_{1}, q_{1}$ are two coprime polynomials with $\operatorname{deg} p_{1} \geq 1$. 
In fact, if $\Delta_{c} R \equiv 0$, then $\frac{q(z+c)}{p(z+c)} \equiv \frac{q(z)}{p(z)}$. Since $\operatorname{deg} p \geq 1$, there exists a finite complex number $z_{0}$ such that $p\left(z_{0}\right)=0$. It follows that $p\left(z_{0}+c\right)=0, p\left(z_{0}+2 c\right)=0, \ldots, p\left(z_{0}+(\operatorname{deg} p+1) c\right)=0$, a contradiction. Thus we prove $\Delta_{c} R \not \equiv 0$. Obviously, there exists a finite complex number $z_{1}$ such that $p\left(z_{1}+c\right)=0, p\left(z_{1}\right) \neq 0, q\left(z_{1}+c\right) \neq 0$, thus we obtain $\operatorname{deg} p_{1} \geq 1$.

Next, using mathematical induction, we obtain

$$
\Delta_{c}^{n} R=\Delta_{c}\left(\Delta_{c}^{n-1} R\right)=\frac{q_{n}(z)}{p_{n}(z)} \not \equiv 0,
$$

where $p_{n}, q_{n}$ are two coprime polynomials with $\operatorname{deg} p_{n} \geq 1$. Thus the proof of the lemma is completed.

\section{Proof of Theorem 1}

Proof Now we prove (1.1). Suppose that $\lambda(f(z)-R(z))<\rho(f)$. Next we will prove $\lambda\left(\Delta_{c}^{n} f(z)-\right.$ $R(z))=\rho(f)$.

Since $\lambda(f(z)-a)<\rho(f)$, and $R$ is a nonconstant rational function, we have

$$
\frac{f(z)-R(z)}{f(z)-a}=\alpha(z) e^{p(z)}
$$

where $\alpha(\not \equiv 0, \infty)$ is a meromorphic function such that $\rho(\alpha)<\rho(f)$, and $p$ is a nonconstant polynomial with $\operatorname{deg} p=\rho(f)$.

Hence we have

$$
T(r, \alpha)=S\left(r, e^{p}\right), \quad T(r, f)=T\left(r, e^{p}\right)+S(r, f) .
$$

By (3.1), we obtain

$$
f(z)=a+\frac{a-R(z)}{\alpha(z) e^{p(z)}-1} .
$$

Thus we have

$$
\begin{aligned}
\Delta_{c}^{n} f(z) & =\Delta_{c}^{n}(f(z)-a) \\
& =\sum_{i=0}^{n}(-1)^{i} C_{n}^{i}(f(z+(n-i) c)-a) \\
& =\sum_{i=0}^{n}(-1)^{i} C_{n}^{i} \frac{a-R(z+(n-i) c)}{\alpha(z+(n-i) c) e^{p(z+(n-i) c)}-1} \\
& =\frac{\sum_{i=0}^{n}(-1)^{i} C_{n}^{i}(a-R(z+(n-i) c)) \Pi_{j \neq i}^{n}\left(\alpha(z+(n-j) c) e^{p(z+(n-j) c)}-1\right)}{\Pi_{i=0}^{n}\left(\alpha(z+(n-i) c) e^{p(z+(n-i) c)}-1\right)} \\
& =\frac{B_{m}(z)\left(e^{p(z)}\right)^{m}+B_{m-1}(z)\left(e^{p(z)}\right)^{m-1}+\cdots+B_{1}(z) e^{p(z)}+B_{0}(z)}{A_{n+1}(z)\left(e^{p(z)}\right)^{n+1}+A_{n}(z)\left(e^{p(z)}\right)^{n}+\cdots+A_{1}(z) e^{p(z)}+(-1)^{n+1}},
\end{aligned}
$$

where $A_{1}(z), A_{2}(z), \ldots, A_{n+1}(z), B_{0}(z), B_{1}(z), \ldots, B_{m}(z)$ are small functions of $e^{p(z)}$, and $A_{n+1}(z)=\alpha(z) \alpha(z+c) e^{p(z+c)-p(z)} \cdots \alpha(z+n c) e^{p(z+n c)-p(z)} \not \equiv 0, B_{m}(z) \not \equiv 0, B_{0}(z)=(-1)^{n+1} \times$ $\Delta_{c}^{n} R(z), m \leq n$ 
It follows from (3.4) that $\Delta_{c}^{n} f \not \equiv 0$. Suppose that $\Delta_{c}^{n} f \equiv 0$. Next we consider two cases.

Case 1. $e^{p(z+n c)-p(z+(n-i) c)}-\frac{\alpha(z+(n-i) c)}{\alpha(z+n c)} \not \equiv 0, i=1,2, \ldots, n$. Then, by (3.4) and Nevanlinna's second fundamental theorem $[1,17,18]$, we obtain

$$
\begin{aligned}
T\left(r, e^{p}\right)= & T\left(r, \frac{\alpha(z+n c) e^{p(z+n c)}}{\alpha(z+n c) e^{p(z+n c)-p(z)}}\right) \\
\leq & T\left(r, \alpha(z+n c) e^{p(z+n c)}\right)+S\left(r, e^{p}\right) \leq N\left(r, \alpha(z+n c) e^{p(z+n c)}\right) \\
& +N\left(r, \frac{1}{\alpha(z+n c) e^{p(z+n c)}-1}\right)+N\left(r, \frac{1}{\alpha(z+n c) e^{p(z+n c)}}\right)+S\left(r, e^{p}\right) \\
\leq & N\left(r, \frac{1}{\alpha(z+n c) e^{p(z+n c)}-1}\right)+S\left(r, e^{p}\right) \\
= & N\left(r, \frac{1}{\alpha(z+n c) e^{p(z+n c)}-1}, \Delta_{c}^{n} f=0\right)+S\left(r, e^{p}\right) \\
\leq & \sum_{i=1}^{n} N\left(r, \frac{1}{\left(\alpha(z+n c) e^{p(z+n c)}-1\right)-\left(\alpha(z+(n-i) c) e^{p(z+(n-i) c)}-1\right)}\right)+S\left(r, e^{p}\right) \\
= & \sum_{i=1}^{n} N\left(r, \frac{1}{\alpha(z+n c) e^{p(z+n c)}-\alpha(z+(n-i) c) e^{p(z+(n-i) c)}}\right)+S\left(r, e^{p}\right) \\
\leq & \sum_{i=1}^{n} N\left(r, \frac{1}{e^{p(z+n c)-p(z+(n-i) c)}-\frac{\alpha(z+(n-i) c)}{\alpha(z+n c)}}\right)+S\left(r, e^{p}\right) \\
\leq & \sum_{i=1}^{n}\left[T\left(r, e^{p(z+n c)-p(z+(n-i) c)}\right)+T\left(r, \frac{\alpha(z+(n-i) c)}{\alpha(z+n c)}\right)\right]+S\left(r, e^{p}\right) \\
= & S\left(r, e^{p}\right)
\end{aligned}
$$

which is a contradiction.

Case 2. $e^{p(z+n c)-p(z+(n-i) c)}-\frac{\alpha(z+(n-i) c)}{\alpha(z+n c)} \equiv 0$, for some $i \in\{1,2, \ldots, n\}$. If $\operatorname{deg} p \geq 2$, then by $\rho(\alpha)<\rho(f)=\operatorname{deg} p$ and Lemma 1 we get

$$
\rho\left(\frac{\alpha(z+(n-i) c)}{\alpha(z+n c)}\right) \leq \rho(\alpha)-1+\frac{\rho(f)-\rho(\alpha)}{2}<\rho(f)-1=\rho\left(e^{p(z+n c)-p(z+(n-i) c)}\right)
$$

which is a contradiction. Hence $\operatorname{deg} p=1$. So, $\rho(\alpha)<1$. Let $p(z)=A z+B$, then we have $\alpha(z+(n-i) c)=e^{i A c} \alpha(z+n c)$. Without loss of generality, we assume that $i=1$, then we obtain

$$
\alpha(z)=e^{A c} \alpha(z+c)
$$

We claim that $\alpha \neq \infty$. Suppose that there exists $z_{0}$ such that $\alpha\left(z_{0}\right)=\infty$, without loss of generality, let $z_{0}=0$, then we deduce that for all positive integers $j, \alpha(j c)=\infty$. Thus for sufficiently large $r$, and $2 n|c| \leq r<(2 n+1)|c|$, we have

$$
\begin{aligned}
T(r, \alpha) & \geq N(r, \alpha)=\int_{0}^{r} \frac{n(t, \alpha)-n(0, \alpha)}{t} d t+n(0, \alpha) \log r \\
& \geq \sum_{j=1}^{2 n-1} j \int_{j|c|}^{(j+1)|c|} \frac{d t}{t}=\sum_{j=1}^{2 n-1} j \log \left(1+\frac{1}{j}\right)
\end{aligned}
$$




$$
\begin{aligned}
& \geq \sum_{j=1}^{2 n-1} j \log \left(1+\frac{1}{2 n-1}\right)=n \log \left(1+\frac{1}{2 n-1}\right)^{2 n-1} \\
& \geq n \log 2>\frac{\log 2}{4|c|} r .
\end{aligned}
$$

It follows that $\rho(\alpha) \geq 1$, which is a contradiction. Similarly, we obtain $\alpha \neq 0$. Thus we deduce that $\alpha$ is a nonzero constant $C$. By (3.1), we obtain

$$
f(z)=a+\frac{a-R(z)}{C e^{A z+B}-1}=a+\frac{a-R(z)}{D e^{A z}-1}, \quad D=C e^{B} \neq 0
$$

If $R=q / p$ be a nonconstant rational function, where $p, q$ are two polynomials with $\operatorname{deg} p \geq 1$, or $R$ is a polynomial with $\operatorname{deg} R \geq n$, then, by Lemma 6, (3.5) and (3.7), we obtain $\Delta_{c}^{n} f \not \equiv 0$. If $R$ is a polynomial with $1 \leq \operatorname{deg} R<n$, and $e^{A c} \neq 1$, then, by (3.7) and some computation, we deduce that $\Delta_{c}^{n} f \not \equiv 0$.

In fact, by (3.4) and (3.7) we have

$$
\begin{aligned}
\Delta_{c}^{n} f(z) & =\sum_{i=0}^{n}(-1)^{i} C_{n}^{i} \frac{a-R(z+(n-i) c)}{D e^{A(z+(n-i) c)}-1} \\
& =\frac{\sum_{i=0}^{n}(-1)^{i} C_{n}^{i}(a-R(z+(n-i) c)) \Pi_{j \neq i}^{n}\left(D e^{A(z+(n-j) c)}-1\right)}{\Pi_{i=0}^{n}\left(D e^{p(z+(n-i) c)}-1\right)} \\
& =\frac{\left[D^{n}\left(e^{A c}\right)^{1+2+\cdots+(n-1)} \sum_{i=0}^{n}(-1)^{i} C_{n}^{i}(a-R(z+(n-i) c))\left(e^{A c}\right)^{i}\right]\left(e^{A z}\right)^{n}+\cdots}{A_{n+1}(z)\left(e^{A z}\right)^{n+1}+A_{n}(z)\left(e^{A z}\right)^{n}+\cdots+A_{1}(z) e^{A z}+(-1)^{n+1}} \\
& =\frac{\left[-D^{n}\left(e^{A c}\right)^{1+2+\cdots+(n-1)} a_{s} \sum_{i=0}^{n}(-1)^{i} C_{n}^{i}\left(e^{A c}\right)^{i} z^{s}+\cdots\right]\left(e^{A z}\right)^{n}+\cdots}{A_{n+1}(z)\left(e^{A z}\right)^{n+1}+A_{n}(z)\left(e^{A z}\right)^{n}+\cdots+A_{1}(z) e^{A z}+(-1)^{n+1}} \\
& =\frac{\left[-D^{n}\left(e^{A c}\right)^{1+2+\cdots+(n-1)} a_{s}\left(e^{A c}-1\right)^{n} z^{s}+\cdots\right]\left(e^{A z}\right)^{n}+\cdots}{A_{n+1}(z)\left(e^{A z}\right)^{n+1}+A_{n}(z)\left(e^{A z}\right)^{n}+\cdots+A_{1}(z) e^{A z}+(-1)^{n+1}}
\end{aligned}
$$

where $R(z)=a_{s} z^{s}+a_{s-1} z^{s-1}+\cdots+a_{1} z+a_{0}, 1 \leq s=\operatorname{deg} R<n$. It follows from $a_{s} \neq 0, D \neq$ $0, e^{A c}-1 \neq 0$ that $\Delta_{c}^{n} f \not \equiv 0$.

Hence by the above discussion we prove that $\Delta_{c}^{n} f \not \equiv 0$. Set

$$
\begin{aligned}
& B\left(e^{p}\right)=B_{m}\left(e^{p}\right)^{m}+B_{m-1}\left(e^{p}\right)^{m-1}+\cdots+B_{1} e^{p}+B_{0} \\
& A\left(e^{p}\right)=A_{n+1}\left(e^{p}\right)^{n+1}+A_{n}\left(e^{p}\right)^{n}+\cdots+A_{1} e^{p}+(-1)^{n+1} .
\end{aligned}
$$

By the method of successive division, there exist $D\left(e^{p}\right), E\left(e^{p}\right), F\left(e^{p}\right), G\left(e^{p}\right), H\left(e^{p}\right)$ satisfying

$$
\begin{aligned}
& A\left(e^{p}\right)=D\left(e^{p}\right) E\left(e^{p}\right), \quad B\left(e^{p}\right)=D\left(e^{p}\right) F\left(e^{p}\right), \\
& E\left(e^{p}\right) G\left(e^{p}\right)+F\left(e^{p}\right) H\left(e^{p}\right) \equiv 1,
\end{aligned}
$$

where

$$
\begin{aligned}
& D\left(e^{p}\right)=D_{l}\left(e^{p}\right)^{l}+D_{l-1}\left(e^{p}\right)^{l-1}+\cdots+D_{1} e^{p}+D_{0}, \\
& E\left(e^{p}\right)=E_{n+1-l}\left(e^{p}\right)^{n+1-l}+E_{n-l}\left(e^{p}\right)^{n-l}+\cdots+E_{1} e^{p}+E_{0},
\end{aligned}
$$




$$
F\left(e^{p}\right)=F_{m-l}\left(e^{p}\right)^{m-l}+E_{m-1-l}\left(e^{p}\right)^{m-1-l}+\cdots+F_{1} e^{p}+F_{0}
$$

and $D_{0}, D_{1}, \ldots, D_{l}, E_{0}, E_{1}, \ldots, E_{n+1-l}, F_{0}, F_{1}, \ldots, F_{m-l}$ are small functions of $e^{p}$, and $D_{l} \not \equiv 0$, $E_{n+1-l} \not \equiv 0, F_{m-l} \not \equiv 0, E_{0} D_{0}=(-1)^{n+1}, 0 \leq l \leq m$.

So by (3.5) and (3.8), we have

$$
\begin{aligned}
\Delta_{c}^{n} f(z)-R(z) & =\frac{B\left(e^{p}\right)}{A\left(e^{p}\right)}-R(z)=\frac{B\left(e^{p}\right)-R(z) A\left(e^{p}\right)}{A\left(e^{p}\right)} \\
& =\frac{F\left(e^{p}\right) D\left(e^{p}\right)-R(z) E\left(e^{p}\right) D\left(e^{p}\right)}{E\left(e^{p}\right) D\left(e^{p}\right)}, \\
& =\frac{F\left(e^{p}\right)-R(z) E\left(e^{p}\right)}{E\left(e^{p}\right)} .
\end{aligned}
$$

It follows that

$$
\left[F_{0}-R(z) E_{0}\right] D_{0}=B_{0}-R(z) A_{0}=(-1)^{n+1}\left[\Delta_{c}^{n} R(z)-R(z)\right] .
$$

By Lemma 5 , we know that $\Delta_{c}^{n} R-R \not \equiv 0$. Thus we deduce that $F_{0}-R(z) E_{0} \not \equiv 0$. By this and (3.9)-(3.10), we obtain

$$
N\left(r, \frac{1}{\Delta_{c}^{n} f(z)-R(z)}\right)=N\left(r, \frac{1}{F\left(e^{P}\right)-R(z) E\left(e^{p}\right)}\right)+S\left(r, e^{p}\right) .
$$

By (3.10) and Lemma 2, we obtain

$$
T\left(r, \Delta_{c}^{n} f(z)\right)=(n+1-l) T\left(r, e^{p}\right)+S(r, f) .
$$

Let $F\left(e^{p}\right)-R(z) E\left(e^{p}\right)=R_{n+1-l}\left(e^{p}\right)^{n+1-l}+\cdots+R_{1}(z) e^{p}+R_{0}(z)$, then $R_{n+1-l}=-R(z) E_{n+1-l}(z)(\not \equiv$ $0), R_{n-l}(z), \ldots, R_{1}(z), R_{0}(z)=F_{0}-R(z) E_{0}(\not \equiv 0)$ are small functions of $e^{p}$. Thus by Lemma 2 , Lemma 4 and Nevanlinna's second fundamental theorem, we obtain

$$
\begin{aligned}
(n+1 & -l) T\left(r, e^{p}\right) \\
= & T\left(r, R_{n+1-l}\left(e^{p}\right)^{n+1-l}+\cdots+R_{1} e^{p}\right) \\
\leq & \bar{N}\left(r, \frac{1}{R_{n+1-l}\left(e^{p}\right)^{n+1-l}+\cdots+R_{1} e^{p}}\right)+\bar{N}\left(r, R_{n+1-l}\left(e^{p}\right)^{n+1-l}+\cdots+R_{1} e^{p}\right) \\
& +\bar{N}\left(r, \frac{1}{R_{n+1-l}\left(e^{p}\right)^{n+1-l}+\cdots+R_{1} e^{p}+R_{0}}\right)+S\left(r, e^{p}\right) \\
\leq & (n-l) T\left(r, e^{p}\right)+\bar{N}\left(r, \frac{1}{R_{n+1-l}\left(e^{p}\right)^{n+1-l}+\cdots+R_{1} e^{p}+R_{0}}\right)+S\left(r, e^{p}\right) .
\end{aligned}
$$

It follows that

$$
T\left(r, e^{p}\right) \leq \bar{N}\left(r, \frac{1}{R_{n+1-l}\left(e^{p}\right)^{n+1-l}+\cdots+R_{1} e^{p}+R_{0}}\right)+S\left(r, e^{p}\right) .
$$

From this together with (3.11) we deduce that

$$
N\left(r, \frac{1}{\Delta_{c}^{n} f(z)-R(z)}\right) \geq T\left(r, e^{p}\right)+S\left(r, e^{p}\right) .
$$


By (3.2), (3.12) and (3.13), we deduce that $\lambda\left(\Delta_{c}^{n} f(z)-R(z)\right)=\rho(f)$. Thus we prove (1.1). Now we prove (1.2). Suppose that

$$
\max \{\lambda(f(z)-R(z)), \lambda(f(z+n c)-R(z))\}<\rho(f) .
$$

Then, by Lemma 3, we have $\lambda(f(z)-R(z-n c))<\rho(f)$. Hence there exist $\varepsilon_{0}>0, T>0$, for $r>T$, we have

$$
\begin{aligned}
& N\left(r, \frac{1}{f-a}\right) \leq r^{\rho(f)-\varepsilon_{0}}, \quad N\left(r, \frac{1}{f-R}\right) \leq r^{\rho(f)-\varepsilon_{0}}, \\
& N\left(r, \frac{1}{f-R(z-n c)}\right) \leq r^{\rho(f)-\varepsilon_{0}} .
\end{aligned}
$$

By the above formulas and Lemma 4, we obtain

$$
\begin{aligned}
T(r, f) & \leq \bar{N}\left(r, \frac{1}{f-a}\right)+\bar{N}\left(r, \frac{1}{f-R}\right)+\bar{N}\left(r, \frac{1}{f-R(z-n c)}\right)+S(r, f) \\
& \leq 3 r^{\rho(f)-\varepsilon_{0}}+O(\log r) .
\end{aligned}
$$

Thus we deduce that $\rho(f) \leq \rho(f)-\varepsilon_{0}$, a contradiction. Hence we prove (1.2).

Next we prove (1.3). Suppose that $\lambda(f(z+n c)-R(z))<\rho(f)$. Then, by Lemma 3, we deduce that $\lambda(f(z)-R(z-n c))<\rho(f)$. In the following, using the same methods as used in the proof of (1.1), we obtain $\lambda\left(\Delta_{c}^{n} f(z)-R(z)\right)=\rho(f)$. Thus we prove (1.3). Therefore, Theorem 1 is proved.

\section{Proof of Theorem 2}

Proof We first prove (2.1). Suppose that $\lambda(f(z)-R(z))<\rho(f)$. Next we prove $\lambda\left(\Delta_{c}^{n} f(z)-\right.$ $R(z))=\rho(f)$.

Since $\lambda\left(\frac{1}{f(z)}\right)<\rho(f)$, and $R$ is a nonconstant rational function, we have

$$
f(z)-R(z)=\alpha(z) e^{p(z)}
$$

where $\alpha$ is a meromorphic function satisfying $\rho(\alpha)<\rho(f)$, and $p$ is a nonconstant polynomial with $\operatorname{deg} p=\rho(f)$.

Hence we have

$$
T(r, \alpha)=S\left(r, e^{p}\right), \quad T(r, f)=T\left(r, e^{p}\right)+S(r, f) .
$$

Thus we have

$$
\begin{aligned}
& \Delta_{c}^{n} f(z)-R(z) \\
& \quad=\Delta_{c}^{n} R(z)+\Delta_{c}^{n}\left(\alpha(z) e^{p(z)}\right)-R(z) \\
& \quad \leq \sum_{i=0}^{n}(-1)^{i} C_{n}^{i}\left(\alpha(z+(n-i) c) e^{p(z+(n-i) c)}-\left(R(z)-\Delta_{c}^{n} R(z)\right)\right.
\end{aligned}
$$




$$
\begin{aligned}
& \leq \sum_{i=0}^{n}(-1)^{i} C_{n}^{i}\left(\alpha(z+(n-i) c) e^{p(z+(n-i) c)-p(z)} e^{p(z)}-\left(R(z)-\Delta_{c}^{n} R(z)\right)\right. \\
& =A(z) e^{p(z)}-\left(R(z)-\Delta_{c}^{n} R(z)\right),
\end{aligned}
$$

where $A(z)=\sum_{i=0}^{n}(-1)^{i} C_{n}^{i}\left(\alpha(z+(n-i) c) e^{p(z+(n-i) c)-p(z)}\right.$.

By (4.2) and Lemma 3, we deduce that $T(r, A)=S(r, f)$.

Since $\Delta_{c}^{n} f(z)$ is a transcendental meromorphic function, it follows that $A \not \equiv 0$. By Lemma $6, R(z)-\Delta_{c}^{n} R(z) \not \equiv 0$. Thus by (4.2), (4.3) and Nevanlinna's second fundamental theorem, we obtain

$$
\begin{aligned}
T\left(r, \Delta_{c}^{n} f\right) & =T\left(r, e^{p}\right)+S(r, f), \\
T\left(r, e^{p}\right) & \leq T\left(r, A e^{p}\right)+T\left(r, \frac{1}{A}\right)+O(1) \\
\leq & N\left(r, A e^{p}\right)+N\left(r, \frac{1}{A e^{p}}\right)+N\left(r, \frac{1}{A e^{p}-\left(R-\Delta_{c}^{n} R\right)}\right)+S\left(e^{p}\right) \\
\leq & N\left(r, \frac{1}{A e^{p}-\left(R-\Delta_{c}^{n} R\right)}\right)+S\left(e^{p}\right) .
\end{aligned}
$$

Thus we obtain

$$
N\left(r, \frac{1}{\Delta_{c}^{n} f-R}\right) \geq T\left(r, e^{p}\right)+S(r, f) .
$$

It follows that $\lambda\left(\Delta_{c}^{n} f(z)-R(z)\right)=\rho(f)$. Thus we prove (2.1).

Now we prove (2.2). Suppose that $\max \{\lambda(f(z)-R(z)), \lambda(f(z+n c)-R(z))\}<\rho(f)$. Then, by Lemma 3, we have $\lambda(f(z)-R(z-n c))<\rho(f)$. Hence there exist $\varepsilon_{0}>0, T>0$, for $r>T$, we have

$$
N(r, f) \leq r^{\rho(f)-\varepsilon_{0}}, \quad N\left(r, \frac{1}{f-R}\right) \leq r^{\rho(f)-\varepsilon_{0}}, \quad N\left(r, \frac{1}{f-R(z-n c)}\right) \leq r^{\rho(f)-\varepsilon_{0}} .
$$

By the above formulas and Lemma 4, we obtain

$$
\begin{aligned}
T(r, f) & \leq \bar{N}(r, f)+\bar{N}\left(r, \frac{1}{f-R}\right)+\bar{N}\left(r, \frac{1}{f-R(z-n c)}\right)+S(r, f) \\
& \leq 3 r^{\rho(f)-\varepsilon_{0}}+O(\log r) .
\end{aligned}
$$

Thus we deduce that $\rho(f) \leq \rho(f)-\varepsilon_{0}$, a contradiction. Hence we prove (2.2).

Next we prove (2.3). Suppose that $\lambda(f(z+n c)-R(z))<\rho(f)$. Then, by Lemma 3, we deduce that $\lambda(f(z)-R(z-n c))<\rho(f)$. In the following, using the same methods as used in the proof of (2.1), we obtain $\lambda\left(\Delta_{c}^{n} f(z)-R(z)\right)=\rho(f)$. Thus we prove (2.3). Theorem 2 is proved.

\section{Conclusion}

In this paper, we have discussed the exponent of convergence of zero points of $f(z)-R(z)$, $f(z+n c)-R(z)$ and $\Delta_{c}^{n} f-R(z)$, where $R(z)$ is a nonconstant rational function. Examples are given to show that all conditions of our results are necessary. Whether the nonconstant rational function can be replaced by a small functions of $f(z)$ is still open. 
Acknowledgements

The authors thank the referee for his/her valuable suggestions to improve the present article.

\section{Funding}

Research supported by the NNSF of China (Grant No. 11701188).

\section{Availability of data and materials}

All data generated or analysed during this study are included in this published article [and its supplementary information files].

\section{Competing interests}

The authors declare that they have no competing interests.

\section{Authors' contributions}

All the authors drafted the manuscript, and read and approved the final manuscript.

\section{Author details}

${ }^{1}$ Department of Mathematics, Hangzhou Dianzi University, Baiyang Road, Hangzhou, China. ${ }^{2}$ Institute of Applied Mathematics, South China Agricultural University, Wushan Road, Guangzhou, China.

\section{Publisher's Note}

Springer Nature remains neutral with regard to jurisdictional claims in published maps and institutional affiliations.

Received: 8 September 2020 Accepted: 29 November 2020 Published online: 09 December 2020

\section{References}

1. Araci, S., Acikgoz, M.: Computation of Nevanlinna characteristic functions derived from generating functions of some special numbers. J. Inequal. Appl. 2018, 128 (2018)

2. Bergweiler, W., Pang, X.C.: On the derivative of meromorphic functions with multiple zeros. J. Math. Anal. Appl. 278(2), 285-292 (2003)

3. Chen, H.Y., Zheng, X.M.: Fixed points of meromorphic functions and their higher order differences and shifts. Open Math. 17,677-688 (2019)

4. Chen, Z.X.: The fixed points and hyper order of solutions of second order complex differential equations. Acta Math Sci. 20(3), 425-432 (2000)

5. Chen, Z.X.: Fixed points of meromorphic functions and of their differences and shifts. Ann. Pol. Math. 109(2), 153-163 (2013)

6. Chen, Z.X., Shon, K.H.: On zeros and fixed points of differences of meromophic function. J. Math. Anal. Appl. 344, $373-383(2008)$

7. Chiang, Y.M., Feng, S.J.: On the Nevanlinna characteristic of $f(z+\eta)$ and difference equations in the complex plane. Ramanujan J. 16(1), 105-129(2008)

8. Chiang, Y.M., Feng, S.J.: On the growth of logarithmic differences, difference quotients and logarithmic derivatives of meromorphic functions. Trans. Am. Math. Soc. 361, 3767-3791 (2009)

9. Chuang, C.T., Yang, C.C.: The Theory of Fixed Points and Factorization of Meromorphic Functions. Beijing University Press, Beijing (1986)

10. Fang, M.L.: A note on a problem of Hayman. Analysis 20(1), 45-49 (2000)

11. Gross, F.: Factorization of Meromorphic Functions. U.S. Government Printing Office, Washington (1972)

12. Halburd, R.G., Korhonen, R., Tohge, K.: Holomorphic curves with shift-invariant hyperplane preimages. Trans. Am. Math. Soc. 366(8), 4267-4298 (2014)

13. Halburd, R.G., Korhonen, R.J.: Difference analogue of the lemma on the logarithmic derivative with applications to difference equations. J. Math. Anal. Appl. 314(2), 477-487 (2006)

14. Halburd, R.G., Korhonen, R.J.: Nevanlinna theory for the difference operator. Ann. Acad. Sci. Fenn., Math. 31(2), 463-478 (2006)

15. Hayman, W.K.: Meromorphic Functions. Clarendon Press, Oxford (1964)

16. Laine, I.: Nevanlinna Theory and Complex Differential Equations. de Gruyter, Berlin (1993)

17. Yang, C.C., Yi, H.X.: Uniqueness Theory of Meromorphic Functions. Kluwer Academic, Dordrecht (2003)

18. Yang, L.: Value Distribution Theory. Springer, Berlin (1993)

19. Zhang, R.R., Chen, Z.X.: Fixed points of meromorphic functions and their differences, divided differences and shifts. Acta Math. Sin. Engl. Ser. 32(10), 1189-1202 (2016) 